\title{
A Motor Vibration Control for Robot Arm with Application to Surgical Tools
}

\author{
Hamid Cheraghi, Farzin Piltan, Nasim Sobhani, Maryam Rahmani and Farzin \\ Matin \\ Intelligent System and Robotic Lab, Iranian Institute of Advance Science and \\ Technology (IRAN SSP), Shiraz/Iran \\ Email: piltan_f@iranssp.com,WWW.IRANSSP.COM/english
}

\begin{abstract}
Multi Degree of Freedom Joints (MDJ) are typically thought to be used for industrial purposes however they are beginning to gain the attention of the medical field. A current application pertains to using the precision and stability of a robot arm with MDJ to assist in minimally invasive surgery. In this research, adaptive sliding mode controls are presented as robust controls for multi degree of freedom joints. The objective of the study is to design controls for actuators without the knowledge of boundary of uncertainties/disturbances by using an adaptive sliding mode control while elucidating the robustness of the adaptive sliding mode control. A sliding mode control provides for ultimate accuracy in the presence of the bounded disturbance/uncertainties, although the sliding mode control also causes chattering. Chattering is undesirable for use with robot medical tools, since it might causes damage to them with a subsequent loss of accuracy and oscillation on end-effectors tools. Such chatter is caused by overestimation of the controller gain. An adaptive sliding mode is proposed as a solution to the problems created by chattering.
\end{abstract}

Keywords: Multi Degree of Freedom Joints, invasive surgery, sliding mode controls, uncertainties, adaptive method, chattering

\section{Introduction}

Surgery is a medical procedure involving an incision in the human body performed to repair damage or arrest disease. In a fully invasive operation, an open incision is made that is large enough for the surgeon to view the internal organs and perform the operation. For example, in open-heart surgery a long incision is made along the sternum, after which the sternum is split and retracted (median sternotomy). These open incisions increase trauma to a patient beyond what is experienced from the actual repair. After the operation, the patient must heal from trauma associated with the repaired organs and from the open incision. This results in long recovery times and opportunities for infection. Endoscopic Surgery, also called Minimally Invasive Surgery (MIS), is a type of operation that has been developed to reduce trauma associated with making these large open incisions. This type of operation involves viewing the operational field on a television monitor by inserting a special camera, called an endoscope, through a small incision in the skin (see Figure 1). To perform the operation," long, thin, manually operated instruments" are inserted through other small incisions called trocars. This type of surgery reduces the size of the open incisions and therefore results in less pain and scarring after surgery, faster recovery times, and less risk of infection. 


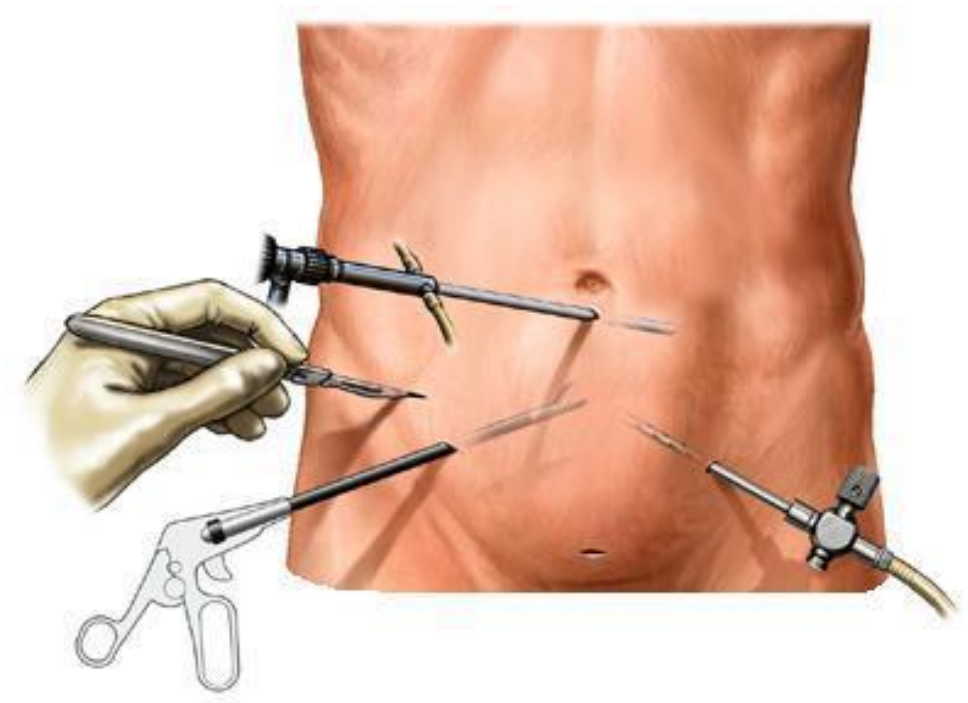

Figure 1. Manual Endoscopic Surgery

Endoscopic surgery does have benefits over fully invasive surgery but there are also several disadvantages associated with performing this type of operation. The two basic disadvantages involve viewing the internal operational field and the surgical tool interface. Using an endoscopic camera, the operational field is viewed on a 2-D television monitor providing only a limited view with no depth perception. Advances in visual technology have provided surgeons with equipment that allow them to view the field in 3 -D however this technology is expensive. Another disadvantage is that the endoscope is operated by a surgical assistant. This requires the surgeon to communicate motion instructions which becomes difficult when giving instructions such as how far to move the endoscope and in which direction. It has also been reported that small tremors from the scope-holding assistant, magnified onto the television monitor, can cause nausea among the surgical team. Disadvantages associated with the surgical tool interface involve the endoscopic tools and the way that the operation is performed. Often the endoscopic tools are heavy, lacking ergonomic design, and do not have the same DOF/dexterity as a human hand (see Figure 2). As mentioned before, these tools are inserted through small incisions in the patient to perform the operation. This creates the fulcrum/lever effect whereas the surgeon is required to transpose each hand motion to get the desired internal motion.

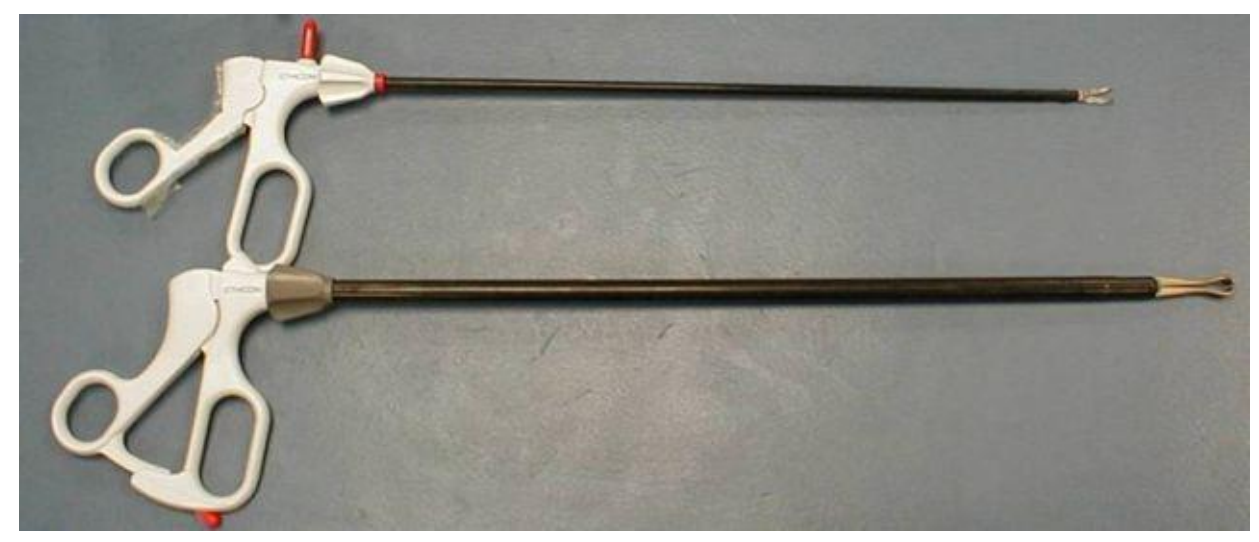

Figure 2. Manual Endoscopic Tools 
In the late 1980's, researchers motivated by the limitations of endoscopic surgery turned to robotic technology to make improvements. Although the idea of using robotics for telepresence surgery was not a new idea at this time, research and development into this concept rapidly progressed around the success of MIS techniques and became possible with advancements in computing power. By 1998, two major companies had developed surgical systems approved by the FDA for commercial use. Computer Motion in Goletta, California developed the Zeus Surgical System and Intuitive Surgical in Sunnyvale, California developed the daVinci Surgical System. Currently only the daVinci is in production due to the merger of Computer Motion and Intuitive Surgical in 2003. One of the significant engineering assistance to surgery is MDA.

Multi degrees of freedom joint (MDJ) is a type of nonlinear joints. These actuators are finding wide use in a number of Industries such as aerospace, Industrial medical and automotive. For high precession trajectory planning and control, it is necessary to replace the actuator system made up of several single-DOF motors connected in series and/or parallel with a single multi-DOF actuator [1-3]. The spherical motor (MDJ) have potential contributions to a wide range of applications such as coordinate measuring, object tracking, material handling, automated assembling, welding, and laser cutting [4]. All these applications require high precision motion and fast dynamic response, which the spherical motor is capable of delivering [5-6]. The spherical motor exhibits coupled, nonlinear and very complex dynamics. The design and implementation of feedback controllers for the motor are complicated. The controller design is further complicated by the orientation-varying torque generated by the spherical motor [7]. One of the significant challenges in control algorithms is a linear behavior controller design for nonlinear systems. Designing an effective and robust control for a nonlinear system with unmodeled dynamics and system disturbances/uncertainties is one of the most significant issues facing control engineers [12]. In the real world, system dynamics are seldom fully known, and more unknown disturbances will arise during the operation. Because there are some uncertainties or unknown disturbances within the system, many engineers have developed a dedicated design control which is insensitive to changes in system dynamics; this dedicated control has become known as a robust control [9-11]. The sliding mode control is an example of a dedicated design control that is used as a robust control [9]. The main feature of the sliding mode control is that it is insensitive to disturbances and uncertainties, if those are bounded.

However, the sliding mode control introduces chattering, which is one of the most significant problems in the field of sliding mode control [9-10]. Overestimating the boundaries of uncertainties and disturbances leads to high sliding mode controller gains and thus increases chattering. Chattering in systems with a sliding mode control is usually caused by the un-modeled dynamics and can be observed as high frequency (but less than infinity) control switching. The chattering effect in control results in oscillations in the sliding variable dynamics. These oscillations prevent sliding variables from being constrained to zero, which results in the degradation of the accuracy of sliding variable stabilization. As a solution to this issue, a sliding mode control with gain adaptation has been proposed, because of its ability to reduce the chattering; it retains the main properties of a sliding mode control but can also control a system with matched bounded disturbances, in which the bounds are unknown [11-17].

This paper examines a sliding mode control with a gain adaptation. This paper shows that the sliding mode control with gain adaptation is a better strategy to design successful control models for use in the presence of the unknown bounded disturbances or uncertainties with unknown boundaries, since the adaptive gain sliding mode control mitigates chattering. The structure of the paper is as follows: Section 2, is served as an introduction to the dynamic of three degrees of freedom spherical motor. Part 3, introduces and describes the methodology algorithm. Section 4 
presents the simulation results and discussion of this algorithm applied to three degrees of freedom spherical motor and the final section describe the conclusion.

\section{Theory}

Dynamic and Kinematics Formulation of Spherical Motor: Dynamic modeling of spherical motors is used to describe the behavior of spherical motor such as linear or nonlinear dynamic behavior, design of model based controller such as pure sliding mode controller which design this controller is based on nonlinear dynamic equations, and for simulation. The dynamic modeling describes the relationship between motion, velocity, and accelerations to force/torque or current/voltage and also it can be used to describe the particular dynamic effects (e.g., inertia, coriolios, centrifugal, and the other parameters) to behavior of system. Spherical motor has nonlinear and uncertain dynamic parameters 3 degrees of freedom (DOF) motor [15-17].

The equation of a spherical motor governed by the following equation:

$$
H(q)\left[\begin{array}{c}
\ddot{\alpha} \\
\ddot{\beta} \\
\ddot{\gamma}
\end{array}\right]+\mathbf{B}(\boldsymbol{q})\left[\begin{array}{c}
\dot{\alpha} \dot{\beta} \\
\dot{\alpha} \dot{\gamma} \\
\dot{\boldsymbol{\beta}} \dot{\gamma}
\end{array}\right]+\boldsymbol{C}(\boldsymbol{q})\left[\begin{array}{c}
\dot{\alpha}^{2} \\
\dot{\boldsymbol{\beta}}^{2} \\
\dot{\gamma}^{2}
\end{array}\right]=\left[\begin{array}{c}
\tau_{x} \\
\tau_{y} \\
\tau_{z}
\end{array}\right]
$$

Where $\tau$ is actuation torque, $\mathrm{H}(\mathrm{q})$ is a symmetric and positive define inertia matrix, $\mathrm{B}(\mathrm{q})$ is the matrix of coriolios torques, $\mathrm{C}(\mathrm{q})$ is the matrix of centrifugal torques.

This is a decoupled system with simple second order linear differential dynamics. In other words, the component $\ddot{q}$ influences, with a double integrator relationship, only the variable $q_{i}$, independently of the motion of the other parts. Therefore, the angular acceleration is found as to be:

$$
\ddot{\boldsymbol{q}}=\boldsymbol{H}^{-\mathbf{1}}(\boldsymbol{q}) .\{\boldsymbol{\tau}-\{\boldsymbol{B}+\boldsymbol{C}\}\}
$$

This technique is very attractive from a control point of view.

Study of spherical motor is classified into two main groups: kinematics and dynamics. Calculate the relationship between rigid bodies and final part without any forces is called Kinematics. Study of this part is pivotal to design with an acceptable performance controller, and in real situations and practical applications. As expected the study of kinematics is divided into two main parts: forward and inverse kinematics. Forward kinematics has been used to find the position and orientation of task frame when angles of joints are known. Inverse kinematics has been used to find possible joints variable (angles) when all position and orientation of task frame be active.

The main target in forward kinematics is calculating the following function:

$$
\boldsymbol{\Psi}(\boldsymbol{X}, \boldsymbol{q})=\mathbf{0}
$$

Where $\Psi(.) \in R^{n}$ is a nonlinear vector function, $X=\left[X_{1}, X_{2}, \ldots \ldots, X_{l}\right]^{T}$ is the vector of task space variables which generally task frame has three task space variables, three orientation, $q=\left[q_{1}, q_{2}, \ldots, q_{n}\right]^{T}$ is a vector of angles or displacement, and finally $n$ is the number of actuated joints. The Denavit-Hartenberg (D-H) convention is a method of drawing spherical motor free body diagrams. Denvit-Hartenberg (D-H) convention study is necessary to calculate forward kinematics in this motor.

A systematic Forward Kinematics solution is the main target of this part. The first step to compute Forward Kinematics (F.K) is finding the standard D-H parameters. The following steps show the systematic derivation of the standard D-H parameters.

1. Locate the spherical motor

2. Label joints

3. Determine joint rotation $(\theta)$

4. Setup base coordinate frames.

5. Setup joints coordinate frames.

6. Determine $\alpha_{i}$, that $\alpha_{i}$, link twist, is the angle between $Z_{i}$ and $Z_{i+1}$ about an $X_{i}$. 
7. Determine $d_{i}$ and $a_{i}$, that $a_{i}$, link length, is the distance between $Z_{i}$ and $Z_{i+1}$ along $X_{i} . d_{i}$, offset, is the distance between $X_{i-1}$ and $X_{i}$ along $Z_{i}$ axis.

8. Fill up the D-H parameters table. The second step to compute Forward kinematics is finding the rotation matrix $\left(R_{n}^{0}\right)$. The rotation matrix from $\left\{F_{i}\right\}$ to $\left\{F_{i-1}\right\}$ is given by the following equation;

$\boldsymbol{R}_{\boldsymbol{i}}^{\boldsymbol{i}-1}=\boldsymbol{U}_{\boldsymbol{i}\left(\boldsymbol{\theta}_{i}\right)} \boldsymbol{V}_{\boldsymbol{i}\left(\boldsymbol{\alpha}_{\boldsymbol{i}}\right)}$

Where $U_{i\left(\theta_{i}\right)}$ is given by the following equation;

$U_{i\left(\theta_{i}\right)}=\left[\begin{array}{ccc}\cos \left(\theta_{i}\right) & -\sin \left(\theta_{i}\right) & 0 \\ \sin \left(\theta_{i}\right) & \cos \left(\theta_{i}\right) & 0 \\ 0 & 0 & 1\end{array}\right]$

and $V_{i\left(\alpha_{i}\right)}$ is given by the following equation;

$$
V_{i\left(\theta_{i}\right)}=\left[\begin{array}{ccc}
1 & 0 & 0 \\
0 & \cos \left(\alpha_{i}\right) & -\sin \left(\alpha_{i}\right) \\
0 & \sin \left(\alpha_{i}\right) & \cos \left(\alpha_{i}\right)
\end{array}\right]
$$

So $\left(R_{n}^{0}\right)$ is given by

$$
R_{n}^{0}=\left(U_{1} V_{1}\right)\left(U_{2} V_{2}\right) \ldots \ldots \ldots\left(U_{n} V_{n}\right)
$$

The final step to compute the forward kinematics is calculate the transformation ${ }_{n}^{0} T$ by the following formulation [3]

$$
{ }_{n}^{0} T={ }_{1}^{0} T \cdot{ }_{2}^{1} T \cdot{ }_{3}^{2} T \ldots \ldots .{ }_{n}^{n-1} T=\left[\begin{array}{cc}
R_{n}^{0} & 0 \\
0 & 1
\end{array}\right]
$$

\section{Methodology}

Sliding Mode Controller Design: To improve the robustness and stability, sliding mode controller is used as reference model controller for adaptive method. Sliding mode controller is an influential nonlinear controller to certain and uncertain systems which it is based on system's dynamic model. The main reason to opt for this controller is its acceptable control performance in wide range and solves two most important challenging topics in control which names, stability and robustness. Sliding mode control theory for control joint of robot manipulator was first proposed in 1978 by Young to solve the set point problem $\left(\dot{\boldsymbol{q}}_{\boldsymbol{d}}=\mathbf{0}\right)$ by discontinuous method in the following form;

$$
\boldsymbol{\tau}_{(q, t)}= \begin{cases}\boldsymbol{\tau}_{\boldsymbol{i}}^{+}(q, t) & \text { if } \boldsymbol{S}_{\boldsymbol{i}}>0 \\ \boldsymbol{\tau}_{\boldsymbol{i}}^{-}(q, t) & \text { if } \boldsymbol{S}_{i}<0\end{cases}
$$

where $\boldsymbol{S}_{\boldsymbol{i}}$ is sliding surface (switching surface), $\boldsymbol{i}=\mathbf{1}, 2, \ldots \ldots, \boldsymbol{n}$ for $n$-DOF joint, $\boldsymbol{\tau}_{\boldsymbol{i}}(\boldsymbol{q}, \boldsymbol{t})$ is the $\boldsymbol{i}^{\boldsymbol{t h}}$ torque of joint. Sliding mode controller is divided into two main sub controllers:

- Corrective control $\left(\boldsymbol{U}_{\boldsymbol{c}}\right)$

- Equivalent controller $\left(\boldsymbol{U}_{\boldsymbol{e q}}\right)$.

Discontinues controller causes an acceptable tracking performance at the expense of very fast switching. Conversely in this theory good trajectory following is based on fast switching, fast switching is caused to have system instability and chattering phenomenon. Fine tuning the sliding surface slope is based on nonlinear equivalent part. Design a robust controller for multi-DOF-joints is essential because these joints have highly nonlinear dynamic parameters. Consider a nonlinear single input dynamic system is defined by:

$x^{(n)}=f(\vec{x})+b(\vec{x}) u$

Where $\mathrm{u}$ is the vector of control input, $x^{(\boldsymbol{n})}$ is the $\boldsymbol{n}^{\text {th }}$ derivation of $\boldsymbol{x}$, $\boldsymbol{x}=\left[\boldsymbol{x}, \dot{\boldsymbol{x}}, \ddot{\boldsymbol{x}}, \ldots, \boldsymbol{x}^{(\boldsymbol{n}-1)}\right]^{\boldsymbol{T}}$ is the state vector, $\boldsymbol{f}(\boldsymbol{x})$ is unknown or uncertainty, and $\boldsymbol{b}(\boldsymbol{x})$ is of known sign function. The main goal to design this controller is train to the desired state; $\boldsymbol{x}_{\boldsymbol{d}}=\left[\boldsymbol{x}_{\boldsymbol{d}}, \dot{\boldsymbol{x}}_{\boldsymbol{d}}, \ddot{\boldsymbol{x}}_{\boldsymbol{d}}, \ldots, \boldsymbol{x}_{\boldsymbol{d}}^{(\boldsymbol{n}-\mathbf{1})}\right]^{\boldsymbol{T}}$, and trucking error vector is defined by:

$$
\widetilde{x}=x-x_{d}=\left[\widetilde{x}, \ldots, \widetilde{x}^{(n-1)}\right]^{T}
$$


A simple solution to get the sliding condition when the dynamic parameters have uncertainty is the switching control law:

$$
U_{C}=\widehat{U}-K(\vec{x}, t) \cdot \operatorname{sgn}(s)
$$

where the switching function $\mathbf{s g n}(\mathbf{S})$ is defined as

$\operatorname{sgn}(s)= \begin{cases}1 & s>0 \\ -1 & s<0 \\ 0 & s=0\end{cases}$

and the $\boldsymbol{K}(\overrightarrow{\boldsymbol{x}}, \boldsymbol{t})$ is the positive constant.

Based on above discussion, the sliding mode control law for multi-DOF-joints is written as:

$\boldsymbol{U}=\boldsymbol{U}_{\text {eq }}+\boldsymbol{U}_{\boldsymbol{c}}$

where, the model-based component $\boldsymbol{U}_{\boldsymbol{e q}}$ is the nominal dynamics of systems and calculated as follows:

$U_{e q}=\left[H^{-1}(q)\left(\mathbf{B}(q)\left[\begin{array}{c}\dot{\alpha} \dot{\beta} \\ \dot{\alpha} \dot{\gamma} \\ \dot{\beta} \dot{\gamma}\end{array}\right]+C(q)\left[\begin{array}{c}\dot{\alpha}^{2} \\ \dot{\beta}^{2} \\ \dot{\gamma}^{2}\end{array}\right]\right)+\dot{S}\right] H(q)$

and $\boldsymbol{U}_{\boldsymbol{c}}$ is computed as;

$U_{c}=K \cdot \operatorname{sgn}(S)$

The sliding mode control of multi-DOF-joint is calculated as;

$\left[\begin{array}{c}\widehat{\tau_{\alpha}} \\ \widehat{\tau}_{\beta} \\ \widehat{\tau_{\gamma}}\end{array}\right]=\left[H^{-1}(q)\left(\mathbf{B}(q)\left[\begin{array}{c}\dot{\alpha} \dot{\beta} \\ \dot{\alpha} \dot{\gamma} \\ \dot{\beta} \dot{\gamma}\end{array}\right]+C(q)\left[\begin{array}{c}\dot{\alpha}^{2} \\ \dot{\beta}^{2} \\ \dot{\gamma}^{2}\end{array}\right]\right)+\dot{S}\right] H(q)+K \cdot \operatorname{sgn}(S)$

The lyapunov formulation can be written as follows [22],

$V=\frac{1}{2} S^{T}$. H.S

the derivation of $V$ can be determined as,

$\dot{V}=\frac{1}{2} S^{T} . \dot{H} \cdot S+S^{T} H \dot{S}$

the dynamic equation of multi-DOF actuator can be written based on the sliding surface as

$H \dot{S}=-V S+H \dot{S}+B+C$

it is assumed that

$S^{T}(\dot{H}-2 B+C) S=0$

by substituting (20) in (21)

$\dot{V}=\frac{1}{2} S^{T} \dot{H} S-S^{T} B+C S+S^{T}(H \dot{S}+B+C S)=S^{T}(H \dot{S}+B+C S)$

suppose the control input is written as follows

$\widehat{U}=U_{\text {Nonltnear }}+\widehat{U_{c}}=\left[\widehat{H^{-1}}(B+C)+\dot{S}\right] \widehat{H}+K \cdot \operatorname{sgn}(S)+B+C S$

by replacing

$\dot{V}=S^{T}\left(H \dot{S}+B+C-\widehat{H} \dot{S}-\widehat{B+C S}-K \operatorname{sgn}(S)=S^{T}(\widetilde{H} \dot{S}+\widetilde{B+C S}-K \operatorname{sgn}(S))\right.$

and

$|\widetilde{\boldsymbol{H}} \dot{\boldsymbol{S}}+\widetilde{\boldsymbol{B + C}} \boldsymbol{S}| \leq|\widetilde{\boldsymbol{H}} \dot{\boldsymbol{S}}|+|\widetilde{\boldsymbol{B}+\boldsymbol{C}} \boldsymbol{S}|$

the Lemma equation in multi-DOF actuator can be written as follows

$K_{u}=[|\widetilde{H} \dot{S}|+|B+C S|+\eta]_{i}, i=1,2,3,4, \ldots$

and finally;

$\dot{V} \leq-\sum_{i=1}^{n} \eta_{i}\left|S_{i}\right|$ 
A super-twisting control is a continuous second-order sliding mode control. This approach is effective for relative degree 1 . The advantage of this control technique is that it eliminates chattering. In other words, a super-twisting control stabilizes the sliding variables robustly at 0 in finite time for any bounded disturbance.

The sliding variable dynamics for a super-twisting control is given to be as follows:

$\dot{\sigma}=\boldsymbol{\varphi}+\boldsymbol{g u}$

Where $|\dot{\varphi}| \leq C, 0 \leq K_{m} \leq g \leq K_{M}$

For the sliding variable dynamics given in Equation (28), the super-twisting control is introduced as:

$$
\left\{\begin{array}{c}
u=-c|\sigma|^{\frac{1}{2}} \operatorname{sign}(\sigma)+\omega \\
\omega=-\dot{b} \cdot \operatorname{sign}(\sigma)
\end{array}\right.
$$

Where

$$
c=1.5 \sqrt{C}, \quad b=1.1 C, \quad|\dot{\varphi}| \leq C
$$

This super-twisting control creates the compensated dynamics of sliding variables as follows:

$$
\sigma+c|\sigma|^{\frac{1}{2}} \operatorname{sign}(\sigma)+b \int \operatorname{sign}(\sigma) d t=\varphi
$$

In sliding mode, $b \int \operatorname{sign}(\sigma) d t$ is equal to $\varphi$. In order to achieve asymptotic convergence of the state variables, the super-twisting control drives both sigma and the first derivative of sigma to zero in finite time and keeps them at 0 thereafter.

To improve robustness adaptive gain super-twisting is used. Adaptive control has an important role in modern control systems. During operation, many controlled processes experience abrupt or continuous parameter variations, varying external conditions and, in some occasions, alternations of operating modes. When all aforementioned causes of non-uniform system behavior are not excessive, then they are usually well handled with standard feedback controllers. But, when that is not the case, then standard feedback controllers cannot maintain the desired control quality and some sort of adaptation to the new situation in the process are needed. Adaptation may be used for the purpose of improving system dynamics or to reduce system sensitivity to parameter variations. Based on above discussion, compute the best value of sliding surface slope coefficient has played important role to improve system's tracking performance especially the system parameters are unknown or uncertain. This problem is solved by tuning the surface slope coefficient $(\boldsymbol{\lambda})$ of the sliding mode controller continuously in real-time. In this methodology, the system's performance is improved with respect to the classical sliding mode controller. Based on super twisting control, to adjust the sliding surface slope coefficient we define $\hat{f}(x \mid \lambda)$ as the tuning.

$\hat{f}(x \mid \lambda)=\lambda^{T} \zeta(x)$

If minimum error $\left(\lambda^{*}\right)$ is defined by;

$\lambda^{*}=\arg \min [(\operatorname{Sup} \mid \hat{f}(x \mid \lambda)-f(x))]$

where $\lambda^{T}$ is adjusted by an adaption law and this law is designed to minimize the error's parameters of $\lambda-\lambda^{*}$. adaption law in sliding mode controller is used to adjust the sliding surface slope coefficient.

The Lyapunov function in this design is defined as

$V=\frac{1}{2} S^{T} H S+\frac{1}{2} \sum_{J=1}^{M} \frac{1}{\gamma_{s j}} \phi^{T} \cdot \phi_{j}$

where $\gamma_{s j}$ is a positive coefficient, $\boldsymbol{\phi}=\boldsymbol{\theta}^{*}-\boldsymbol{\theta}, \boldsymbol{\theta}^{*}$ is minimum error and $\theta$ is adjustable parameter. Since $\dot{M}-2 V$ is skew-symetric matrix; 
$S^{T} H \dot{S}+\frac{1}{2} S^{T} \dot{H} S=S^{T}(H \dot{S}+V S)$

the controller formulation is defined by

$\tau=\widehat{\boldsymbol{H}} \ddot{\boldsymbol{q}}_{r}+\widehat{\boldsymbol{V}} \dot{\boldsymbol{q}}_{r}-\lambda \boldsymbol{S}-\boldsymbol{K}$

According to

$\boldsymbol{H}(\boldsymbol{q}) \ddot{\boldsymbol{q}}+\boldsymbol{V}(\boldsymbol{q}, \dot{\boldsymbol{q}}) \dot{\boldsymbol{q}}=\widehat{\boldsymbol{H}} \ddot{\boldsymbol{q}}_{r}+\widehat{\boldsymbol{V}} \dot{\boldsymbol{q}}_{r}-\lambda \boldsymbol{S}-\boldsymbol{K}$

Since $\dot{\boldsymbol{q}}_{r}=\dot{\boldsymbol{q}}-\boldsymbol{S}$ and $\ddot{\boldsymbol{q}}_{r}=\ddot{\boldsymbol{q}}-\dot{\boldsymbol{S}}$

$H \dot{S}+(V+\lambda) S=\Delta f-K$

$\boldsymbol{H} \dot{S}=\Delta f-K-V S-\lambda S$

$\dot{V}=S^{T} H \dot{S}+\frac{1}{2} S^{T} \dot{H} S+\sum_{J=1}^{M} \frac{1}{\gamma_{s j}} \phi^{T} \cdot \dot{\phi}_{j}$

$\dot{V}=S^{T}(H \dot{S}+V S)+\sum_{J=1}^{M} \frac{1}{\gamma_{s j}} \phi^{T} \cdot \dot{\phi}_{j}$

Based on

$\dot{V}=S^{T}(\Delta f-K-V S-\lambda S+V S)+\sum_{J=1}^{M} \frac{1}{\gamma_{s j}} \phi^{T} \cdot \dot{\phi}_{j}$

where $\Delta f=[H(q) \ddot{q}+V(q, \dot{q}) \dot{q}]-\sum_{l=1}^{M} \theta^{T} \zeta(x)$

$\dot{V}=\sum_{J=1}^{M}\left[S_{j}\left(\Delta f_{\mathrm{j}}-K_{j}\right)\right]-S^{T} \lambda S+\sum_{J=1}^{M} \frac{1}{\gamma_{s j}} \phi^{T} \cdot \dot{\phi}_{j}$

suppose $K_{j}$ is defined as follows

$K_{j}=\frac{\sum_{l=1}^{M} \theta_{j}^{l}\left[\mu_{A}\left(S_{j}\right)\right]}{\sum_{l=1}^{M}\left[\mu_{A}\left(S_{j}\right)\right]}=\theta_{j}^{T} \zeta_{j}\left(S_{j}\right)$

Where $\zeta_{j}\left(S_{j}\right)=\left[\zeta_{j}^{1}\left(S_{j}\right), \zeta_{j}^{2}\left(S_{j}\right), \zeta_{j}^{3}\left(S_{j}\right), \ldots ., \zeta_{j}^{M}\left(S_{j}\right)\right]^{T}$

$\zeta_{j}^{1}\left(S_{j}\right)=\frac{\mu_{(A)}^{l}\left(S_{j}\right)}{\left.\sum_{i} \mu_{(A)}\right)_{j}^{l}\left(S_{j}\right)}$

where $\mu_{(x i)}$ is evaluation algorithm.

according to;

$\dot{V}=\sum_{J=1}^{M}\left[S_{j}\left(\Delta f_{\mathrm{j}}-\theta^{T} \zeta\left(S_{j}\right)\right]-S^{T} \lambda S+\sum_{J=1}^{M} \frac{1}{\gamma_{s j}} \phi^{T} . \dot{\phi}_{j}\right.$ 
Based on $\boldsymbol{\phi}=\boldsymbol{\theta}^{*}-\boldsymbol{\theta} \rightarrow \boldsymbol{\theta}=\boldsymbol{\theta}^{*}-\boldsymbol{\phi}$

$\dot{V}=\sum_{J=1}^{M}\left[S_{j}\left(\Delta f_{\mathrm{j}}-\theta^{* T} \zeta\left(S_{j}\right)+\phi^{T} \zeta\left(S_{j}\right)\right]-S^{T} \lambda S+\sum_{J=1}^{M} \frac{1}{\gamma_{s j}} \phi^{T} \cdot \dot{\phi}_{j}\right.$

$\dot{V}=\sum_{J=1}^{M}\left[S_{j}\left(\Delta f_{j}-\left(\theta^{*}\right)^{T} \zeta\left(S_{j}\right)\right]-S^{T} \lambda S+\sum_{J=1}^{M} \frac{1}{\gamma_{s j}} \phi_{j}^{T}\left[\gamma_{s j} . S_{j} \cdot \zeta_{j}\left(S_{j}\right)+\dot{\phi}_{j}\right]\right)$

$\dot{V}=\sum_{j=1}^{m}\left[S_{j} \Delta f_{j}-\left(\left(\theta_{j}^{*}\right)^{T} \zeta_{j}\left(S_{j}\right)\right)\right]-S^{T} \lambda S$

The minimum error is defined by

$\boldsymbol{e}_{\boldsymbol{m} j}=\Delta \boldsymbol{f}_{\boldsymbol{j}}-\left(\left(\boldsymbol{\theta}_{j}^{*}\right)^{\boldsymbol{T}} \boldsymbol{\zeta}_{j}\left(\boldsymbol{S}_{j}\right)\right)$

Therefore $\dot{\boldsymbol{V}}$ is computed as

$$
\begin{aligned}
\dot{V} & =\sum_{\substack{j=1 \\
\leq}}^{m}\left[S_{j} e_{m j}\right]-S^{T} \lambda S \\
& =\sum_{j=1}^{m}\left|S_{j}\right|\left|e_{m j}\right|-S^{T} \lambda S \\
& =\sum_{j=1}^{m}|| e_{m j} \mid-\lambda_{j} S_{j}^{2} \\
& =S_{j} \mid\left(\left|e_{m j}\right|-\lambda_{j} S_{j}\right)
\end{aligned}
$$

The minimum approximation error $\left(e_{m j}\right)$ is very small.

if $\lambda_{j}=\alpha$ that $\alpha\left|S_{j}\right|>\boldsymbol{e}_{m j}\left(\boldsymbol{S}_{j} \neq \mathbf{0}\right) \quad$ then $\dot{V}<0$ for $\left(\boldsymbol{S}_{j} \neq \mathbf{0}\right)$

\section{Results and Discussion}

Proposed adaptive controller and sliding mode controller are compared. This simulation is used to control position of three degrees of freedom spherical motor without and with external disturbance. The simulation was implemented in MATLAB/SIMULINK environment.

To evidence the power of proposed method; this design is compared to command. Regarding to Figure 3; sliding mode controller and proposed method have the same accuracy performance. Sliding mode controller is a fast and limit robust controller and in certain condition it has an acceptable accuracy performance. Regarding to tracking accuracy, rise time in proposed method is about 0.4 second and in sliding mode controller is about 0.6 second. In certain condition, proposed method has $1.3 \%$ overshoot and sliding mode has $4.9 \%$ overshoot. In this state sliding mode controller has a little fluctuations (chattering) compare with proposed method. 


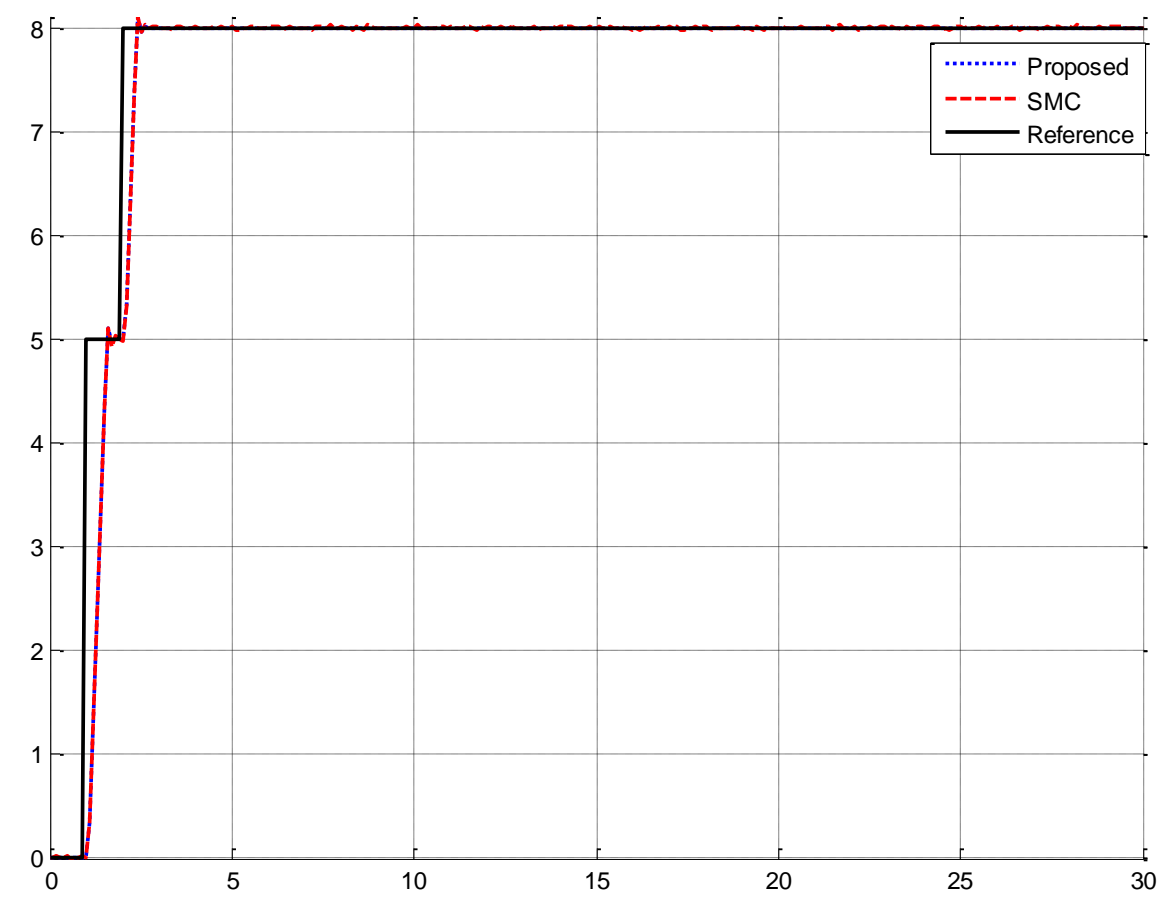

Figure 3. Comparison between Two Types Controller

Figure 4 shows the command and track error joint variables in proposed method and SMC.

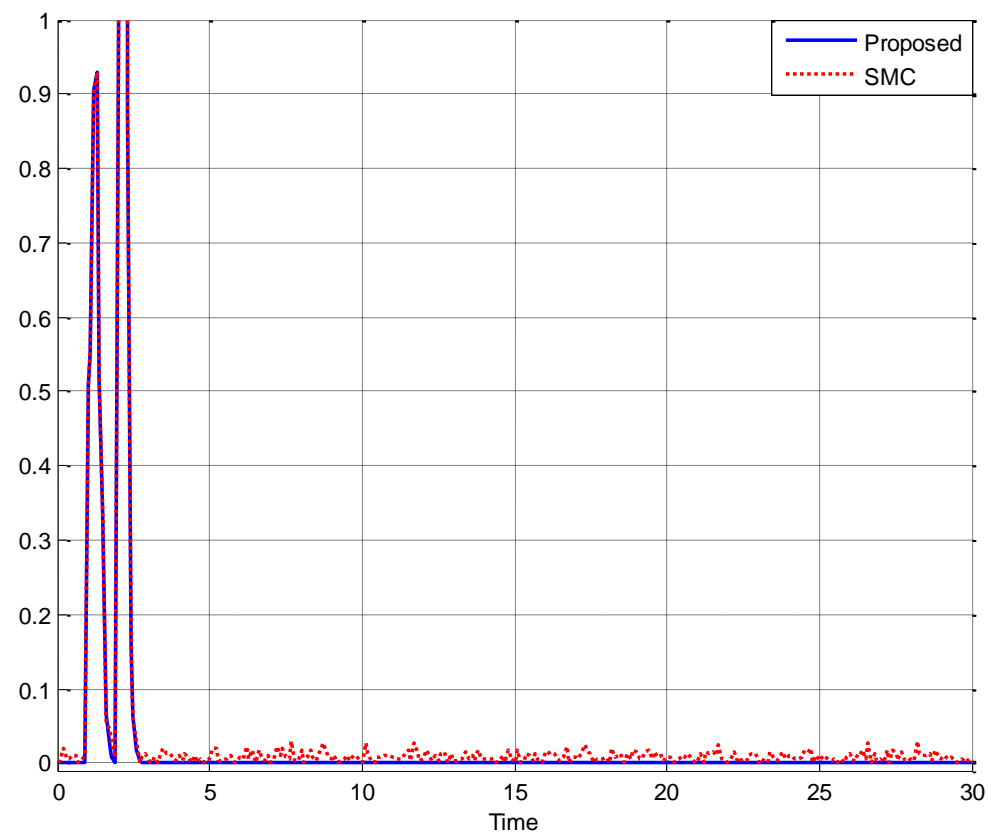

Figure 4. Tracking Error Performance 
Regarding to Figure 4, proposed method tracking error is enough good compare to SMC. SMC has fluctuations and adaptive method can improve the error performance and reduce the RMS error near to the zero. Regarding to Figure 5, torque performance is test in proposed method and compared with sliding mode controller. According to this design, proposed method is more suitable to this design and SMC has fluctuations. Based on the following Figure, proposed method can reduce the energy consumption against to SMC.

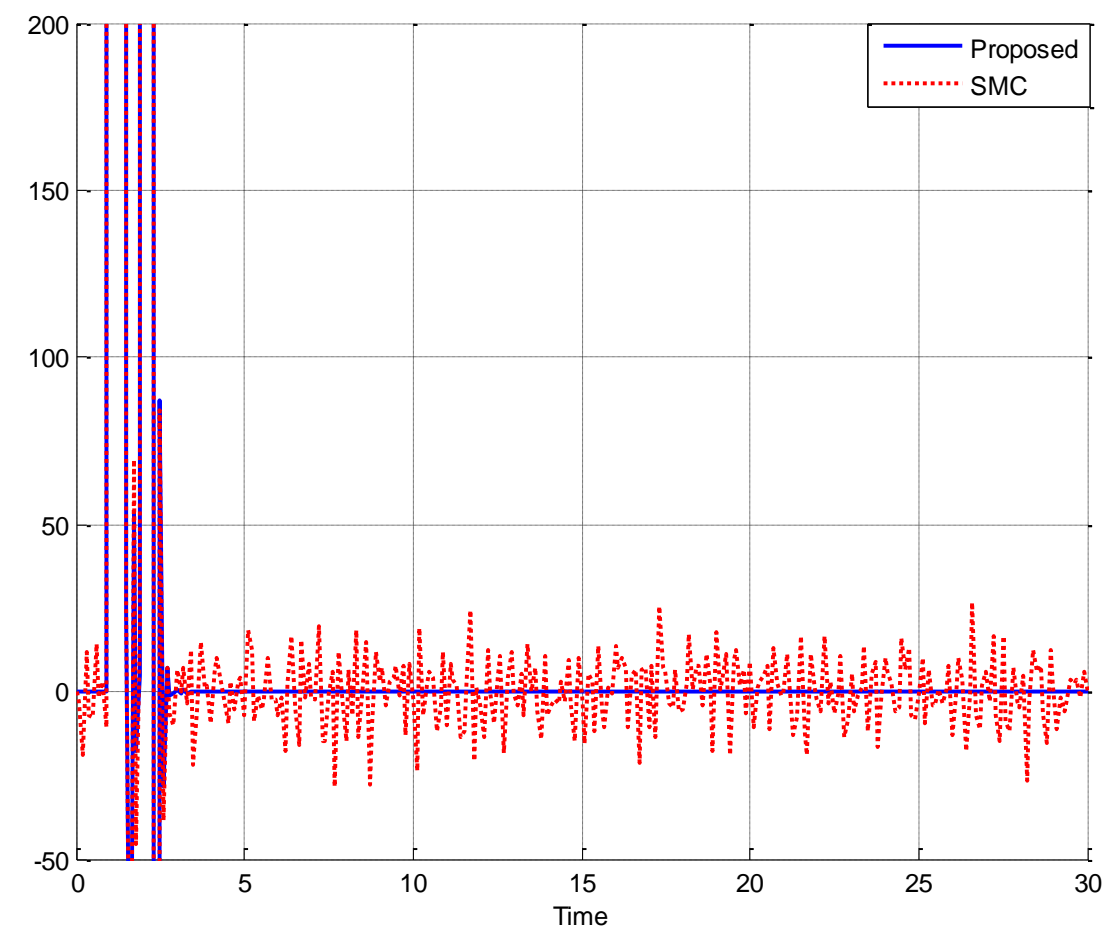

Figure 5. Energy Consumption

\section{Conclusion}

Regarding to this research, gain scheduling sliding mode controller is used as robust controller to improve the performance of multi degree of freedom joint. In robot assistant surgical tools, stability and robustness are two most important factors. To improve the robust factors as well stability, sliding mode adaptive methodology based on gain scheduling methodology is used. Regarding to test and result part, proposed adaptive control is more robust than pure sliding mode controller. This method has power to eliminate the chattering, reduce the overshoot and undershoot, reduce the rise time eliminate the fluctuation in presence of uncertainty. Based on result, proposed methodology can reduce the energy consumption in certain and uncertain condition against to conventional sliding mode controller.

\section{Acknowledgments}

The authors would like to thank the anonymous reviewers for their careful reading of this paper and for their helpful comments. This work was supported by the Iranian Institute of Advance Science and Technology Program of Iran under grant no. 2013Persian Gulf-2A.

Iranian center of Advance Science and Technology (IRAN SSP) is one of the independent research centers specializing in research and training across of Control and 
Automation, Electrical and Electronic Engineering, and Mechatronics \& Robotics in Iran. At IRAN SSP research center, we are united and energized by one mission to discover and develop innovative engineering methodology that solve the most important challenges in field of advance science and technology. The IRAN SSP Center is instead to fill a long standing void in applied engineering by linking the training a development function one side and policy research on the other. This center divided into two main units:

- Education unit

- Research and Development unit

\section{References}

[1] G. I. Vachtsevanos, K. Davey and K. M. Lee, "Development of a Novel Intelligent Robotic Manipulator", IEEE Control System Magazine, (1987), pp. 9-15.

[2] K. Davey, G. I. Vachtsevanos and R. Powers, "An analysis of Fields and Torques in Spherical Induction Motors”, IEEE Transactions on Magnetics, Vol. MAG-23, (1987), pp. 273-282.

[3] A. Foggia, E. Oliver and F. Chappuis, "New Three Degrees of Freedom Electromagnetic Actuator", Conference Record -lAS Annual Meeting, vol. 35, (1988), New York.

[4] K. M. Lee, G. Vachtsevanos and C.-K. Kwan, "Development of a Spherical Wrist Stepper Motor", Proceedings of the IEEE International Conference on Robotics and Automation, Philadelphia, PA, (1988) April 26-29.

[5] K. M. Lee and I. Pei, "Kinematic Analysis of a Three Degree-of-Freedom Spherical Wrist Actuator", The Fifth International Conference on Advanced Robotics, Italy, (1991).

[6] I. Wang, G. Jewel and D. Howe, "Modeling of a Novel Spherical Pennanent Magnet Actuator", Proceedings of IEEE International Conference on Robotics and Automation, Albuquerque, New Mexico, (1997), pp. 1190-1195.

[7] I. Wang, G. Jewel and D. Howe, "Analysis, Design and Control of a Novel Spherical Pennanent Magnet Actuator", IEEE Proceedings on Electrical Power Applications, vol. 154, no. 1, (1998).

[8] G. S. Chirikjian and D. Stein, "Kinematic Design and Commutation of a Spherical Stepper Motor", IEEEIASME Transactions on Mechatronics, Piscataway, New Jersey, vol. 4, no. 4, pp. 342-353, (1999) December.

[9] K. Kahlen and R. W. de Doncker, "CW'l'ent Regulators for Multi-phase Pennanent Magnet Spherical Machines”, Industry Applications Conference Record of the IEEE, vol. 3, (2000), pp. 2011-2016.

[10] K. M. Lee, I. Pei and U. Gilboa, "On the Development of a Spherical Wrist Actuator", Proceedings of the 16th NSF Conference on Manufacturing Systems Research, Tempe AZ, (1990) January 8-12.

[11] C. Yang and Y. S. Back, "Design and Control of the 3-dcgn:es of freedom actuator by Controlling the Electromagnetic Force", IEEE Transactions on Magnetics, (1999) May, pp. 3607-3609.

[12] A. Siahbazi, A. Barzegar, M. Vosoogh, A. M. Mirshekaran and S. Soltani, "Design Modified Sliding Mode Controller with Parallel Fuzzy Inference System Compensator to Control of Spherical Motor", IJISA, vol. 6, no. 3, (2014), pp. 12-25, DOI: 10.5815/ijisa.2014.03.02.

[13] M. Yaghoot, F. Piltan, M. Esmaeili, M. A. Tayebi and M. Piltan, "Design Intelligent Robust Model-base Sliding Guidance Controller for Spherical Motor", IJMECS, vol. 6, no. 3, (2014), pp. 61-72, DOI: 10.5815/ijmecs.2014.03.08.

[14] F. Matin, F. Piltan, H. Cheraghi, N. Sobhani and M. Rahmani, "Design Intelligent PID like Fuzzy Sliding Mode Controller for Spherical Motor", IJIEEB, vol. 6, no. 2, (2014), pp. 53-63, DOI: 10.5815/ijieeb.2014.02.07.

[15] A. Barzegar, F. Piltan, M. Vosoogh, A. M. Mirshekaran and A. Siahbazi, "Design Serial Intelligent Modified Feedback Linearization like Controller with Application to Spherical Motor", IJITCS, vol. 6, no. 5, (2014), pp. 72-83, DOI: 10.5815/ijitcs.2014.05.10.

[16] M. Shamsodini, F. Piltan, S. Rahbar, E. Pooladi and H. Davarpanah, "Design New PID like Fuzzy CTC Controller: Applied to Spherical Motor", IJMECS, vol. 6, no. 5, (2014), pp. 60-68, DOI: 10.5815/ijmecs.2014.05.08.

[17] M. Rahmani, F. Piltan, F. Matin, H. Cheraghi and N. Sobhani, "Design Intelligent System Compensator to Computed Torque Control of Spherical Motor", IJISA, vol. 6, no. 8, (2014), pp. 87-96, DOI: 10.5815/ijisa.2014.08.10.

[18] M. Piltan, F. Piltan, M. Yaghoot, S. Rahbar and M. A. Tayebi, "Design High-Efficiency Intelligent PID like Fuzzy Backstepping Controller for Three Dimension Motor”, IJIEEB, vol. 6, no. 4, (2014), pp. 5262, DOI: 10.5815/ijieeb.2014.04.08. 


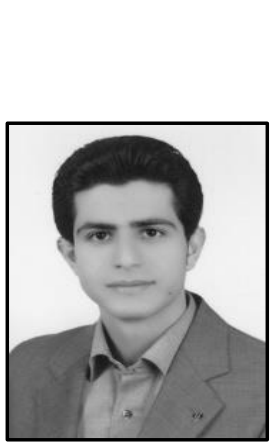

\begin{abstract}
Authors
Hamid Cheraghi, has been working at "Design High Precision and Fast Dynamic Controller for Multi-Degrees of Freedom Actuator for Experimental Research and Education" project at Iranian Institute of Advance Science and Technology, Sanaat Kade Sabz Passargad Research Center (IRAN SSP) as "Student researcher" of a research team composed of 21 researchers since July 2013 to date.
\end{abstract}

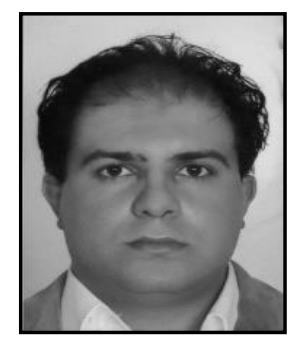

Farzin Piltan, was born on 1975, Shiraz, Iran. In 2004 he is jointed Institute of Advance Science and Technology, Research and Development Center, IRAN SSP. Now he is a dean of Intelligent Control and Robotics Lab. He is led of team (47 researchers) to design and build of nonlinear control of industrial robot manipulator for experimental research and education and published about 54 Papers in this field since 2010 to 2012, team supervisor and leader (9 researchers) to design and implement intelligent tuning the rate of fuel ratio in internal combustion engine for experimental research and education and published about 17 Journal papers since 2011 to 2013, team leader and advisor (34 researchers) of filtering the hand tremors in flexible surgical robot for experimental research and education and published about 31 journal papers in this field since 2012 to date, led of team (21 researchers) to design high precision and fast dynamic controller for multi-degrees of freedom actuator for experimental research and education and published about 7 journal papers in this field since 2013 to date, led of team ( 22 researchers) to research of full digital control for nonlinear systems (e.g., Industrial Robot Manipulator, IC Engine, Continuum Robot, and Spherical Motor) for experimental research and education and published about 4 journal papers in this field since 2010 to date and finally led of team (more than 130 researchers) to implementation of Project Based-Learning project at IRAN SSP research center for experimental research and education, and published more than 110 journal papers since 2010 to date. In addition to 7 textbooks, Farzin Piltan is the main author of more than 115 scientific papers in refereed journals. $\mathrm{He}$ is editorial review board member for 'international journal of control and automation (IJCA), Australia, ISSN: 2005-4297; 'International Journal of Intelligent System and Applications (IJISA)', Hong Kong, ISSN:2074-9058; 'IAES international journal of robotics and automation, Malaysia, ISSN:2089-4856; 'International Journal of Reconfigurable and Embedded Systems', Malaysia, ISSN:2089-4864. His current research interests are nonlinear control, artificial control system and applied to FPGA, robotics and artificial nonlinear control and IC engine modeling and control. 

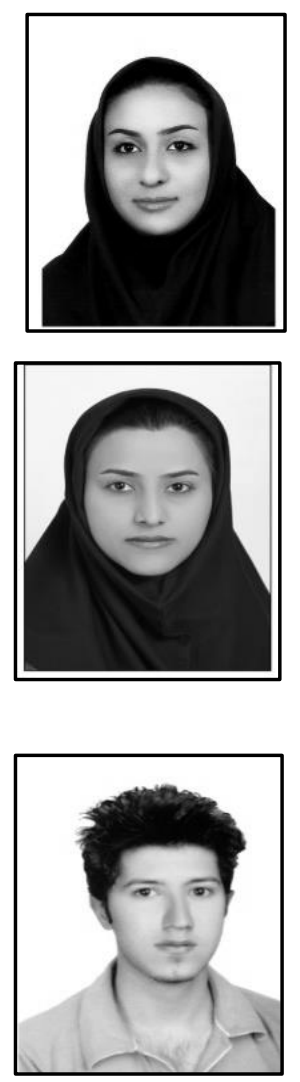

Nasim Sobhani, has been working at "Design High Precision and Fast Dynamic Controller for Multi-Degrees of Freedom Actuator for Experimental Research and Education" project at Iranian Institute of Advance Science and Technology, Sanaat Kade Sabz Passargad Research Center (IRAN SSP) as "Student researcher" of a research team composed of 21 researchers since July. 2013 to date.

Maryam Rahmani, has been working at "Design High Precision and Fast Dynamic Controller for Multi-Degrees of Freedom Actuator for Experimental Research and Education" project at Iranian Institute of Advance Science and Technology, Sanaat Kade Sabz Passargad Research Center (IRAN SSP) as "Student researcher " of a research team composed of 21 researchers since July 2013 to date.

Farzin Matin, has been working at "Design High Precision and Fast Dynamic Controller for Multi-Degrees of Freedom Actuator for Experimental Research and Education" project at Iranian Institute of Advance Science and Technology, Sanaat Kade Sabz Passargad Research Center (IRAN SSP) as "Student researcher " of a research team composed of 21 researchers since July 2013 to date. 